\title{
Introduction to Architectures, Tools, and Methods for Safe and Secure DevOps Software Engineering Minitrack
}

\author{
Damian Tamburri \\ Technical University Eindhoven and Jheronimus \\ Academy of Data Science \\ d.a.tamburri@tue.nl
}

\author{
Willem-Jan Van Den Heuvel \\ Universiteit Van Tilburg and \\ Jheronimus Academy of Data Science \\ wjheuvel@uvt.nl
}

DevOps is recently emerging as a disruptive series of principles and practices that reduce the amount of time between software refactoring and operationally deploying changes. DevOps principles and tools also primarily aim at strengthening the continuity, social, organisational and technical structures maintained by software development and operations engineers in their process of speedily making a design refactoring actionable in operations as well. On one hand, the goal of the tighter continuity along DevOps technical, organizational, and social dimensions is to deliver the software product faster to its production environment, by whatever means, procedures, or tools. On the other hand, establishing and verifying the quality of outcoming software and processes is strained by the "need for speed", especially from the perspective of sensible architecture properties such as Security and Privacy.

This minitrack seeks to shed light over the synergies and challenges in the above mentioned DevOps technical, organizational, and social dimensions, focusing on Security and Privacy concerns from a software engineering perspective. We encourage submissions that cover the above aspects in the scope of:

- Software Architectures that enable or support any of the above dimensions and aspects;

- Tools that speed-up any of the dimensions and aspects or their mutual continuity (e.g., the continuity between Development technical artifacts and Operations technical artifacts);

- Methods to bring about a speed gain in the context of Safe and Secure DevOps pipelines. 